\title{
Mathematical Analysis Of Effect Of Area On The Dynamical Spread Of Measles
}

\author{
S.O. Adewale ${ }^{1}$, I.T. Mohammed ${ }^{2}$ and I.A. Olopade ${ }^{1}$ \\ ${ }^{1}$ Department of Pure and Applied Mathematics, Ladoke Akintola University of Technology Ogbomoso, Nigeria \\ ${ }^{2}$ Department of Mathematics and Statistics Osun State Polytechnic Iree, Nigeria
}

\begin{abstract}
This paper presents a robust compartmental mathematical model of (SVEIR) which incorporated area only. Where this area is the size of the environment where the study is being investigated. It shows that model has a disease free equilibrium which is globally asymptotically stable (GAS). There exists a unique endemic equilibrium point which is locally stable whenever the association threshold quantity $\left(\mathrm{R}_{0}\right)$ exceeds unity i.e. $\mathrm{R}_{0}>1$. We solved the model numerically using Runge-kutta of order four (4). It is shown that as the area is increasing the total number of infected individual is decreasing. This implies that to reduce the spread of measles, measles patients are to be kept separately for treatment so as to reduce the effective contract rate. The results were presented graphically.
\end{abstract}

Keywords: - Measles, Reproduction Number, Equilibrium Point, Habitat Area and Stability.

\section{INTRODUCTION}

Measles is caused by the measles virus. The measles virus is a paramyxovirus of the genus Morbillivirus. It is transmitted by close contact via airborne propagules and spread through droplet transmission from the nose, throat, and mouth of someone who is infected with the virus. These droplets are sprayed out when the infected person coughs or sneezes. Among unimmunized people exposed to the virus, over $90 \%$ will contact the disease. Infection leads to the development of a typical rash. The infected person is highly contagious with the rash appearing four days after the person has been infected. The measles virus can remain in the air (and still be able to cause disease) for up to two hours after an infected person has left a room. Individuals infected with measles virus are believed to be immune for life. Also, individual who have received two doses of vaccine after their first birthday has a $98 \%$ likelihood of being immune, that is, the probability of having measles even when in contact with those infected with measles is above 0.02 , which is very low. Though infants receive some immunity from their mother, this immunity is not complete and they are at increased risk for infection until they receive the vaccinations at 12 to 15 months of age [3, 14].

The infectious period is in the order of a week, after which the hosts recover and develop lifelong immunity. Hosts therefore, are normally infected only once in their lifetime and if the dose of the infectious agent is sufficiently large, this happens at a young age, hence measles is a childhood disease. Although in unvaccinated populations measles is a common disease, infection is not without danger [3, 14].

Individuals at high risk for measles include but not limited to children less than 1 year of age who, though have some immunity passed from their mother, is not $100 \%$ effective;

Individual who have not received the proper vaccination series; individual who received immunoglobulin at the time of measles vaccination and individual immunized from 1963 until 1967 with an older ineffective killed measles vaccine. Mortality rates from measles are often high in tropical Africa because of malnutrition, concurrent infection and inadequate case management. Mortality rates of 5\% and 10\% are common and rates of $20 \%$ have been reported $[3,14]$.

In Nigeria, measles is an important cause of childhood morbidity and mortality. Failure to deliver at least one dose of measles vaccine to all infants remains the main reason for high measles morbidity and mortality as $95 \%$ coverage is required to interrupt measles transmission. The National Program on Immunization aim of reducing measles case fatality to near zero has depended on the adoption and implementation of the WHO four prong strategy; improving routine immunization with at least one dose of measles vaccine at 9 months, providing a second opportunity for measles immunization through supplemental immunization activities, establishing case based surveillance with laboratory confirmation and improving case management (World Health Organization, 2001).

Many physicians and scientists have studied the nature, characteristics, effect and spread of measles in many communities across the globe; these studies are either experimental or theoretical. Similarly, some mathematical modeling studies have been carried out to gain insight into the transmission dynamics and control of measles spread in human population. In this paper, we design a compartmental mathematical model which we sue to investigate the effect of habitat area in the control of the spread of measles in the society. The model designed is 
an extension of some of the models described by previous researchers. The purpose of the current study is to provide a rigorous mathematical analysis of a model for measles spread, which sues standard incidence function for the infection rate and evaluate the conditions at which epidemics will occur and persist using the basic reproduction number concept.

\section{MATHEMATICAL MODEL FORMULATION}

Following $[3,7,8]$, the total homogenously mixing population at time $t$, denoted by $\mathrm{N}(\mathrm{t})$, is sub divided into mutually-exclusive compartments of susceptible $(\mathrm{S}(\mathrm{t}))$, Vaccinated $(\mathrm{V}(\mathrm{t}))$, exposed /latent $(\mathrm{E}(\mathrm{t}))$, intentions $(\mathrm{I}(\mathrm{t}))$, and recovered $(\mathrm{R}(\mathrm{t}))$ individuals, so that

$\mathrm{N}(\mathrm{t})=\mathrm{S}(\mathrm{t})+\mathrm{V}(\mathrm{t})+\mathrm{I}(\mathrm{t})+\mathrm{R}(\mathrm{t})$

The susceptible population is increased by the recruitment of people (either by birth or immigration) into the population; all recruited individuals are assumed to be susceptible at a rate, $\pi$ Also the susceptible population increases by vaccinated individuals. Anyone who has had measles is believed to be immune for life. This population is decreased by infection, which can be acquired following effective contact with infections individuals only at a rate $\lambda$ given by

$$
\lambda=\frac{\beta \eta_{d} I}{N}
$$

In (1) $\beta$ represents the effective contact rate (i.e. contact capable of leaching to measles infection). $\eta_{d}$ is a modification parameter that compares the transmissibility of the diseases. Here also, we assume that $0<\eta_{d}<.1$ Thus the rate of change of the susceptible population is given by

$$
\frac{d S}{d t}=(1-\rho) \pi-\frac{\beta S}{A}-\frac{\lambda S}{A}-\mu S+\omega V
$$

The population of the vaccination individual increases by recruiting individual (either by birth or immigration) and vaccinated individuals into the vaccinated class. The population of vaccinated individual is decrease by the progression of the rate at which vaccine wanes $(\omega)$ and vaccinated individuals is also reduced by natural death (at the rate $\mu$ ). Thus

$$
\frac{d V}{d t}=\rho \pi-\omega V-\mu V
$$

The population of the exposed individual is increased by susceptible individuals whom are infected by those who are infectious per habitat area. The population of the exposed individuals is decreased by exposed individuals whom are infectious and is also reduced by natural death of the exposed individual. Then,

$$
\frac{d E}{d t}=\frac{\beta S}{A}+\frac{\lambda S}{A}-\sigma \mathrm{E}-\mu E
$$

The population of infected individual increased by the exposed individuals whom are infectious. The infected population is decreased by the infected individuals whom are treated and get recovered. The infected individuals are also reduced by those that died of measles and to mention of natural death of measles patient also reduced the population of infected individuals.

Hence,

$$
\frac{d I}{d t}=\sigma E-\tau I-\delta I-\mu I
$$

Recovery here means recovery from diseases. The population of recovered individuals is increase by recovery of infected individuals after treatment. The population is reduced by natural death of recovered individual.

Therefore

$$
\frac{d R}{d t}=\tau I-\mu R
$$

Thus, in summary, the measles dynamics transmission model, is given by the following system of non-linear differential equations,

$$
\begin{aligned}
& \frac{d S}{d t}=(1-\rho) \pi-\frac{\beta S}{A}-\frac{\lambda S}{A}-\mu S+\omega V \\
& \frac{d V}{d t}=\rho \pi-\omega V-\mu V
\end{aligned}
$$




$$
\begin{aligned}
& \quad \frac{d E}{d t}=\frac{\beta S}{A}+\frac{\lambda S}{A}-\sigma E-\mu E \\
& \frac{d \mathrm{I}}{d t}=\sigma \mathrm{E}-\tau \mathrm{I}-\delta \mathrm{I}-\mu \mathrm{I} \\
& \frac{d R}{d t}=\tau \mathrm{I}-\mu R
\end{aligned}
$$

\begin{tabular}{|c|c|}
\hline Parameters & Description. \\
\hline$\rho$ & Fraction of recruitment individuals who are vaccinated. \\
\hline$\pi$ & The recruitment rate of the individuals (either by birth or immigration). \\
\hline$\beta$ & $\begin{array}{l}\text { The rate at which susceptible individuals become infected by those who are } \\
\text { infectious. }\end{array}$ \\
\hline$\mu$ & Natural death rate. \\
\hline$\omega$ & $\begin{array}{l}\text { The rate at which vaccine wanes (i.e. } 1 / \omega \text { is the duration of the loss of immunity } \\
\text { acquired by preventive vaccine or by infectious). }\end{array}$ \\
\hline$\sigma$ & The rate at which exposed individuals becomes infectious. \\
\hline$\tau$ & The rate at which infected individuals are treated and recovered. \\
\hline A & Area per Square meter. \\
\hline$\delta$ & Measles induced mortality rate. \\
\hline$\eta_{d}$ & Modification parameter. \\
\hline
\end{tabular}

Table 1:Description of parameters of the model

\section{ANALYSIS OF THE MODEL}

Theorem 1: The closed set $\mathrm{D}=\left\{(S, V, E, I, R) \in \mathfrak{R}_{+}^{5}: N \leq \frac{\pi}{\mu}\right\}$ is positively invariant and attracting with respect to the model equation (7) above.

Proof: Consider the biologically-feasible region (1), the rate of change of the total population obtained by adding all the equations of the model (7) above is given by

since $N=S+V+E+I+R$

$$
\frac{d N}{d t}=\pi-\mu S-\mu V-\mu E-\mu I-\mu R-\delta I
$$

then

$$
\begin{aligned}
& \frac{d N}{d t}=\frac{d S}{d t}+\frac{d V}{d t}+\frac{d E}{d t}+\frac{d I}{d t}+\frac{d R}{d t} \\
& \frac{d N}{d t}=\pi-\mu S-\mu V-\mu E-\mu I-\mu R-\delta I \\
& =\pi-\mu(S+V+E+I+R)-\delta I \\
& \frac{d N}{d t}=\pi-\mu N-\delta I
\end{aligned}
$$

Therefore, $\frac{d N}{d t}<0$ whenever the sub total population $\mathrm{N}>\frac{\pi}{\mu}$, Hence, for all $\mathrm{t}>0$, all the solutions of the model with the initial conditions in D will remain in $\mathrm{D}$. Thus, the biologically feasible region $\mathrm{D}$ is positivelyinvariant and attracting.

\section{1 Disease Free Equilibrium (DFE)}

The DFE of the modeled equation i.e. (7) can be obtained by setting the right hand side of the model to zero

$$
\varepsilon_{0}=\left(S_{0}, V_{0}, E_{0}, I_{0}, R_{0}\right)=\left\{\frac{A \pi(1-\rho)}{\beta+\mu A}, 0,0,0,0\right\}
$$


The stability of the DFE, $\varepsilon_{0}$ will be analysed using the next generation method. The non-negative matrix $\mathrm{F}$ (of the new infection terms) and the non singular matrix $\mathrm{V}$ ( of the remaining transfer terms) are given respectively

Where

$$
\text { by } F=\left[\begin{array}{cccc}
0 & 0 & 0 & 0 \\
0 & 0 & \frac{\beta \eta_{d}}{A} & 0 \\
0 & 0 & 0 & 0 \\
0 & 0 & 0 & 0
\end{array}\right], \quad V=\left[\begin{array}{cccc}
k_{1} & 0 & 0 & 0 \\
0 & k_{2} & 0 & 0 \\
0 & -\sigma & k_{3} & 0 \\
0 & 0 & -\tau & \mu
\end{array}\right]
$$

$$
k_{1}=(\omega+\mu) ; \quad k_{2}=(\sigma+\mu) ; \quad k_{3}=(\tau+\delta+\mu)
$$

The associated reproduction number, denoted by $R_{0}$ is given by $R_{0}=p\left(F V^{-1}\right)$ where $p$ denote spectral radius (dominant eigenvalue in magnitude) of the next generation matrix $\left(F V^{-1}\right)$

$$
\text { It follows that } R_{0}=\frac{\beta \eta_{d} \sigma}{A k_{2} k_{3}}
$$

where $k_{2}=(\sigma+\mu) ; \quad k_{3}=(\tau+\delta+\mu)$

Hence, the result below follows,

Lemma 1: The DFE of the model equation, given (7), is locally stable if $R_{0}<1$.

The threshold quantity $R_{0}$, is the reproduction number for the model. It measures the average number of new measles infections generated by a single infectious individual in a population where some of the infected individuals have been immunized. The epidemiological implication of this lemma is that measles spread can be effectively controlled in the community (when $\mathrm{R}_{0}<1$ ). If the initial sizes of the sub-population of the model are in the basin of attraction of the diseases-free equilibrium $\left(\varepsilon_{0}=\left(\frac{A \pi(1-\rho)}{\beta+\mu A}, 0,0,0,0\right)\right)$.

Epidemiologically, if $\mathrm{R}_{0}<1$, the disease will dies out in the community and if $\mathrm{R}_{0}>1$, the disease spreads in the population. Hence, the basic reproduction number turned out to be an important factor in determining the transmission dynamics of any infectious diseases.

\subsection{Stability Analysis of the DFE}

Hence, the stability property of the DFE of the model will be explored. At a steady state $S=N^{*}-V-E-I-R$, hence the stability of $\varepsilon_{0}$ can be established by considering the following mass action equivalent of the model given as

$$
\begin{gathered}
\frac{d V}{d t}=\rho \pi-\omega V-\mu V \\
\frac{d E}{d t}=\frac{\beta S}{A}+\frac{\lambda S}{A}-\sigma E-\mu E \\
\frac{d I}{d t}=\sigma E-\tau I-\delta I-\mu I \\
\frac{d R}{d t}=\tau I-\mu R \\
\text { and } \lambda=\frac{\beta \eta_{d} I}{N}
\end{gathered}
$$

Here, the invariance region is given by

$$
D^{*}=\left\{(V, E, I, R) \in \mathfrak{R}_{+}^{4}: V+E+I+R \leq N^{*}\right\}
$$

Theorem 2: The DFE of model (13) given by (11) is global asymptotically stable (GAS) if $R_{0}<1$.

Proof: The equation (13) can be re-written as 
$\left[\begin{array}{l}\frac{d V}{d t} \\ \frac{d E}{d t} \\ \frac{d I}{d t} \\ \frac{d R}{d t}\end{array}\right]=\left(G_{1}-G_{2}\right)\left[\begin{array}{l}V \\ E \\ I \\ R\end{array}\right]$

where the matrices $\mathrm{G}_{1}, \mathrm{G}_{2}$ and $\mathrm{G}_{3}$ are given as:

$$
\begin{aligned}
& G_{1}=\left[\begin{array}{cccc}
0 & 0 & 0 & 0 \\
0 & 0 & \frac{\beta \eta_{d}}{A} & 0 \\
0 & 0 & 0 & 0 \\
0 & 0 & 0 & 0
\end{array}\right] \\
& G_{2}=\left[\begin{array}{cccc}
(\omega+\mu) & 0 & 0 & 0 \\
0 & (\sigma+\mu) & 0 & 0 \\
0 & -\sigma & (\tau+\delta+\mu) & 0 \\
0 & 0 & -\tau & \mu
\end{array}\right] \text { and } \\
& \sigma_{3}=\left[\begin{array}{llll}
x & x & x & x \\
0 & 0 & 0 & 0 \\
0 & 0 & 0 & 0 \\
0 & 0 & 0 & 0
\end{array}\right]
\end{aligned}
$$

Since matrix $\mathrm{G}_{3}$ is non-negative

$$
\left[\begin{array}{l}
\frac{d V}{d t} \\
\frac{d E}{d t} \\
\frac{d I}{d t} \\
\frac{d R}{d t}
\end{array}\right] \leq\left(G_{1}-G_{2}\right)\left[\begin{array}{l}
V \\
E \\
I \\
R
\end{array}\right]
$$

If $R_{0}<1$ then $\left(G_{1} G_{2}^{-1}\right)<1$ (from the local stability result given in Lemma 1), which is equivalent to $\mathrm{G}_{1}-\mathrm{G}_{2}$ having all its eigenvalue in the left-half plane [13]. It follows that the linearized differential inequality system (14) is stable whenever $R_{0}<1$

\section{3 Existence of Endemic Equilibrium Point (EEP)}

In this section, the possible existence and stability of endemic (positive equilibria of the modeled equation (7). where at least one of the infected components of the model is non-zero) will be considered. Let $\varepsilon_{1}=\left(S^{*}, V^{*}, E^{*}, I^{*}, R^{*}\right)$ represents any arbitrary endemic equilibrium of the model equation. Solving the equations of the system at the steady-state goes thus:

$$
\begin{aligned}
& S^{*}=\frac{A \pi(\omega+\mu-\rho \mu)}{(\omega+\mu)(\mu A+\lambda+\beta)} \\
& \Rightarrow \quad V^{*}=\frac{\rho \pi}{\omega+\mu} \\
& E^{*}=\frac{\beta S^{*}+\lambda S^{*}}{A(\sigma+\mu)} \\
& \Rightarrow I^{*}=\frac{\sigma E^{*}}{(\tau+\delta+\mu)}
\end{aligned}
$$

Substitute for $E^{*}$ in (18) to (19)

$$
=I^{*}=\frac{\sigma \beta S^{*}+\sigma \lambda S^{*}}{A(\sigma+\mu)(\tau+\delta+\mu)}=P_{1} S^{*}+P_{2} \lambda^{*} S^{*}
$$




$$
\text { where } \quad P_{1}=\frac{\sigma \beta}{A(\sigma+\mu)(\tau+\delta+\mu)} \quad \text { and } \quad P_{2}=\frac{\sigma}{A(\sigma+\mu)(\tau+\delta+\mu)}
$$

also,

$$
\begin{array}{r}
R^{*}=\frac{\tau I^{*}}{\mu}+\frac{\tau}{\mu}\left(P_{1} S^{*}+P_{2} \lambda^{*} S^{*}\right) \\
R^{*}=\frac{\tau I^{*} P_{1} S^{*}}{\mu}+\frac{\tau P_{2} \lambda^{*} S^{*}}{\mu} \\
\text { recall that, } \lambda=\frac{\beta \eta_{d} I}{N^{*}} \Rightarrow N^{*} \lambda=\beta \eta_{d} I
\end{array}
$$

where $N^{*}=S^{*}+V^{*}+E^{*}+I^{*}+R^{*}$

$$
\begin{aligned}
\lambda \eta S^{*}+ & \frac{\rho \pi}{(\omega+\mu)}+\frac{(\beta+\lambda) S^{*}}{A(\sigma+\mu)}+P_{1} S^{*}+P_{2} \lambda^{*} S^{*}+\frac{\tau}{\mu} P_{1} S^{*}+\frac{\tau}{\mu} P_{2} \lambda^{*} S^{*} \\
& =\beta \eta_{d}\left(P_{1} S^{*}+P_{2} \lambda^{*} S^{*}\right)
\end{aligned}
$$

let

$$
\begin{aligned}
& (\omega+\mu)=k_{1} \quad \text { and } \quad(\sigma+\mu)=k_{2} \text { as before } \\
& \begin{aligned}
\lambda \eta S^{*}+ & \frac{\rho \pi}{k_{1}}+\frac{(\beta+\lambda) S^{*}}{A k_{2}}+P_{1} S^{*}+P_{2} \lambda^{*} S^{*}+\frac{\tau}{\mu} P_{1} S^{*}+\frac{\tau}{\mu} P_{2} \lambda^{*} S^{*} \\
& =\beta \eta_{d}\left(P_{1} S^{*}+P_{2} \lambda^{*} S^{*}\right)
\end{aligned}
\end{aligned}
$$

Subtract $P_{1} S^{*}$ from both sides

$$
\begin{aligned}
\lambda \eta S^{*}+ & \frac{\rho \pi}{k_{1}}+\frac{(\beta+\lambda) S^{*}}{A k_{2}}+P_{2} \lambda^{*} S^{*}+\frac{\tau}{\mu} P_{1} S^{*}+\frac{\tau}{\mu} P_{2} \lambda^{*} S^{*} \\
& =\beta \eta_{d}\left(P_{2} \lambda^{*} S^{*}\right)
\end{aligned}
$$

Divide both sides by $\lambda^{*} S^{*}$

$$
\lambda\left\{1+\frac{\rho \pi}{k_{1}}+\frac{\beta+\lambda}{A k_{2}}+P_{2} \lambda^{*}+\frac{\tau}{\mu} P_{1}+\frac{\tau}{\mu} P_{2} \lambda^{*}=\beta \eta_{d} P_{2} \lambda^{*}\right.
$$

Let $P_{3}=\frac{\rho \pi}{k_{1}}+\frac{\beta+\lambda}{A k_{2}}+P_{2} \lambda^{*}+\frac{\tau}{\mu} P_{1}+\frac{\tau}{\mu} P_{2} \lambda^{*}$

$$
\begin{array}{r}
1+P_{3} \lambda^{*}=\beta \eta_{d} P_{2} \lambda^{*}=R_{0} \\
\lambda^{*}=\frac{R_{0}-1}{P_{3}}
\end{array}
$$

The component of the unique endemic equilibrium $\left(\varepsilon_{1}\right)$ can then be obtained by substituting the unique value of $\lambda^{*}$, given in (24), into the expressions in $(16,18,20)$. Thus the following result has been established.

\subsection{Local stability of EEP}

The local stability of the unique EEP, $\left(\varepsilon_{1}\right)$ will now be explored for the special case where the disease induced mortality is negligible (i.e. $\delta=0$ ), setting $\delta=0$ in the model (2-6) above shows that

$$
\frac{d N(t)}{d t}=\pi-\mu N(t)
$$

Hence, it follows from (23) that $N(t) \rightarrow \frac{\pi}{\mu}=N$ as $t \rightarrow \infty$; further, using the substitution $S=N^{*}-V-E-I-R$ (and noting that $\delta=0$ ) in the model equation (2-6) gives the following reduced model. 


$$
\begin{aligned}
& \frac{d V}{d t}=\rho \pi-(\omega+\mu) V \\
& \begin{aligned}
& \frac{d E}{d t}= \frac{(\beta+\lambda)}{A}\left(N^{*}-V-E-I-R\right)-(\sigma+\mu) E \\
&=\left[\frac{\beta}{A}+\frac{\left(\beta \eta_{d} I\right)}{N A}\right]\left(N^{*}-V-E-I-R\right)-(\sigma+\mu) E \\
&=\frac{\beta}{A}+\frac{\left(1+\eta_{d} I\right)}{N}\left(N^{*}-V-E-I-R\right)-(\sigma+\mu) E \\
& \frac{d I}{d t}=\sigma E-(\tau+\delta+\mu) I \\
& \frac{d R}{d t}=\tau I-\mu R
\end{aligned}
\end{aligned}
$$

So, for the reduced model (26), the associated reproduction number denoted by $R_{0}^{*}$ is given by

$$
R_{0}^{\infty}=\frac{\beta \eta_{d} \sigma}{A(\sigma+\mu)(\tau+\delta+\mu)}
$$

Using the same approach as in Existence of Endemic Equilibrium Point (EEP); it can be shown that the reduced model equation (26) has a unique endemic equilibrium given by $\varepsilon_{1} \mid \delta=0$ whenever $R_{0}^{\infty}>1$. The epidemiological implication of this is that once there is endemic situation of measles in a congested area of a community, the diseases will rapidly spread in that community whenever the associated reproduction number $R_{0}^{\infty}>1$. Hence to avoid the endemic situation we should extend the area where those suffering from measles would be staying. In other words, the associated reproduction number $R_{0}^{\infty}$ less than unity.

\section{NUMERICAL SIMULATIONS}

Numerical Simulations of the dynamic model were carried out by maple 13, using the Rurge-Kutta of order four (4). The set of parameter values in table I were used to investigate the effect of habitat area in the control of the spread of Measles. Five (5) hypothetical cases were considered and in each case, the probability that individuals who are exposed to the diseases will progress fast to infectious class depends on the level of immunity individual has. It is prominent to note here that when measles patient are separated from non-infected (vaccinated or recovered) people and kept in a wider area, it is assumed that they will have herd immunity, (i.e. the level of immunity in a population which prevents epidemics).

We considered five (5) cases as shown in the table below;

\begin{tabular}{|c|c|c|c|c|c|}
\hline Parameters & Case & Case & Case & Case & Case \\
& 1 & 2 & 3 & 4 & 5 \\
\hline$\beta$ & 0.2 & 0.2 & 0.2 & 0.2 & 0.2 \\
\hline$\mu$ & 0.02 & 0.02 & 0.02 & 0.02 & 0.02 \\
\hline$\pi$ & 2000 & 2000 & 2000 & 2000 & 2000 \\
\hline$\sigma$ & 0.09 & 0.09 & 0.09 & 0.09 & 0.09 \\
\hline$\tau$ & 0.8 & 0.8 & 0.8 & 0.8 & 0.8 \\
\hline$\delta$ & 0.04 & 0.04 & 0.04 & 0.04 & 0.04 \\
\hline$\eta_{d}$ & 0.9 & 0.9 & 0.9 & 0.9 & 0.9 \\
\hline$\omega$ & 0.1 & 0.1 & 0.1 & 0.1 & 0.1 \\
\hline$\rho$ & 0.3 & 0.3 & 0.3 & 0.3 & 0.3 \\
\hline $\mathrm{A}$ & 1 & 10 & 100 & 200 & 300 \\
\hline
\end{tabular}

With the following initial conditions:

$\mathrm{S}[0]:=2885 ; \mathrm{V}[0]:=4192 ; \mathrm{E}[0] ;=15784$

I [0]: $=1645 ; \mathrm{R}[0]:=14215$ 


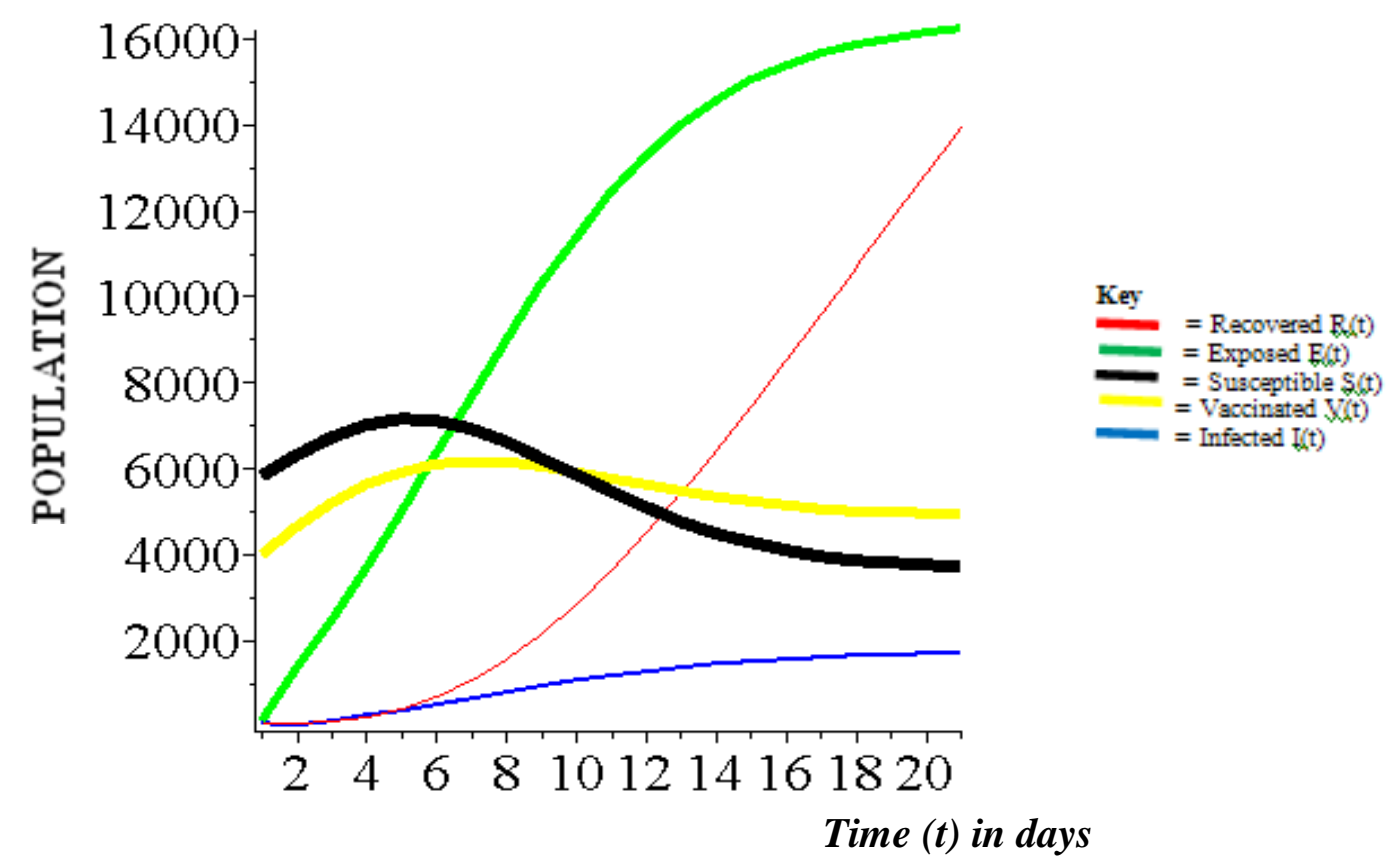

Figure 1: when $\mathbf{A}=1, \beta=0.2, \mathbf{N}=0.02, \pi=2000, \delta=0.04, \sigma=0.09$, $\tau=0.8, \eta_{d}=0.9, \omega=0.1$, and $\rho=0.3$

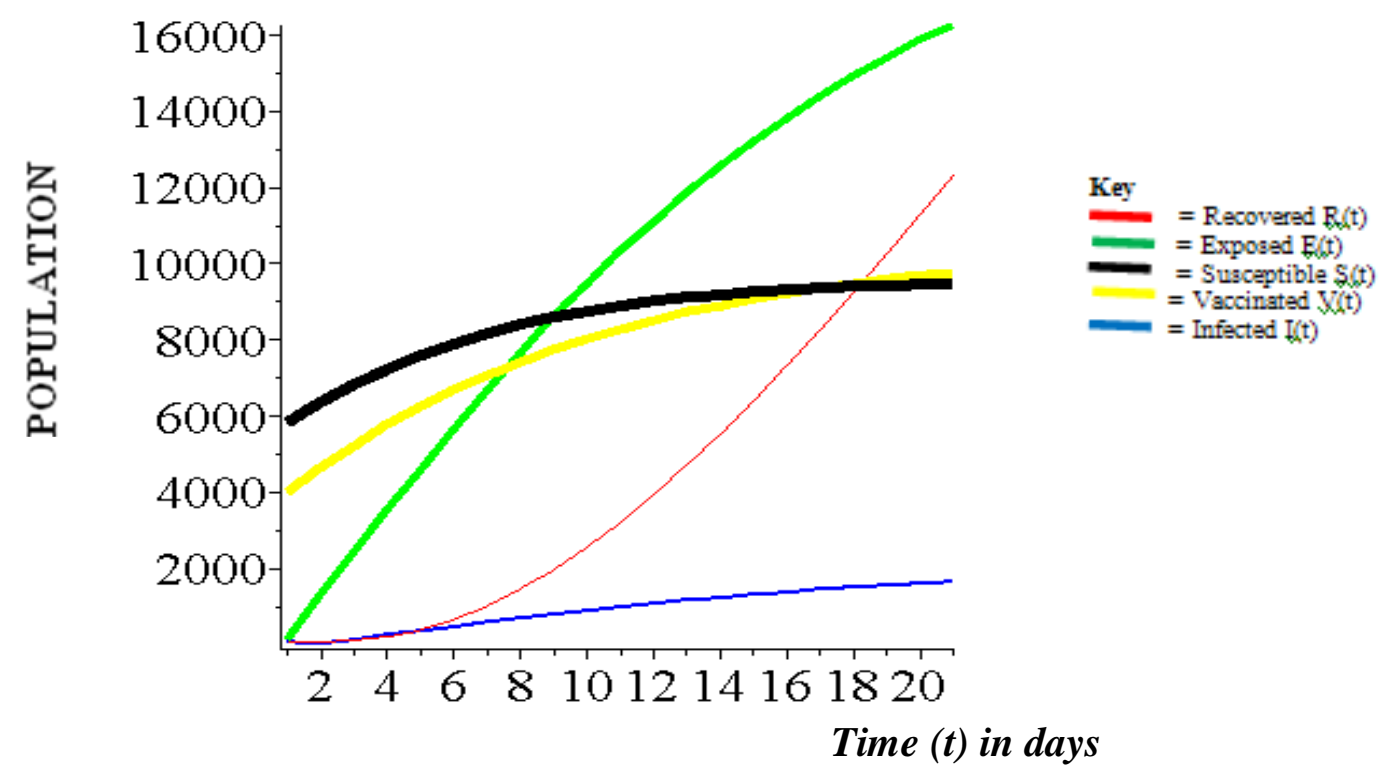

Figure 2: when $\mathrm{A}=10, \beta=0.2, \mathrm{~N}=0.02, \pi=2000, \delta=0.04, \sigma=0.09$, $\tau=0.8, \eta_{d}=0.9, \omega=0.1$ and $\rho=0.3$ 


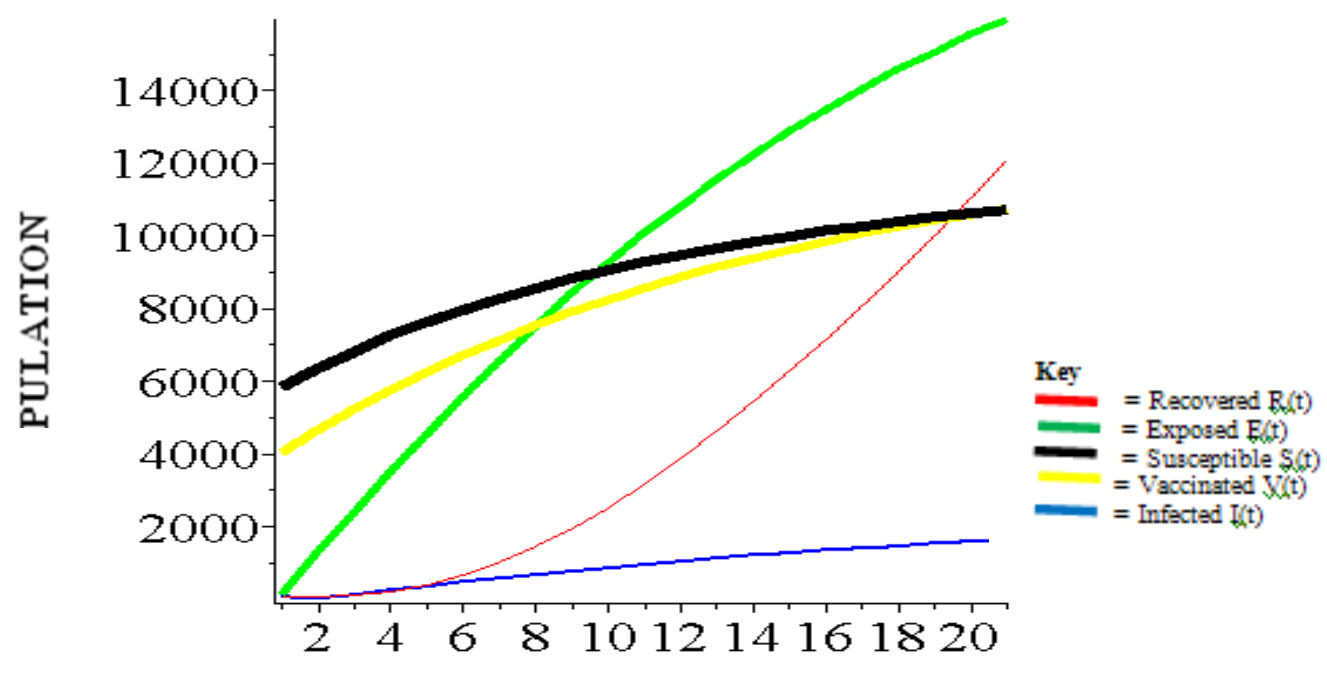

Time $(t)$ in davs

Figure 3: when $\mathbf{A}=100, \beta=0.2, \mathbf{N}=0.02, \pi=2000, \delta=0.04, \sigma=0.09$, $\tau=0.8, \eta_{d}=0.9, \omega=0.1$ and $\rho=0.3$

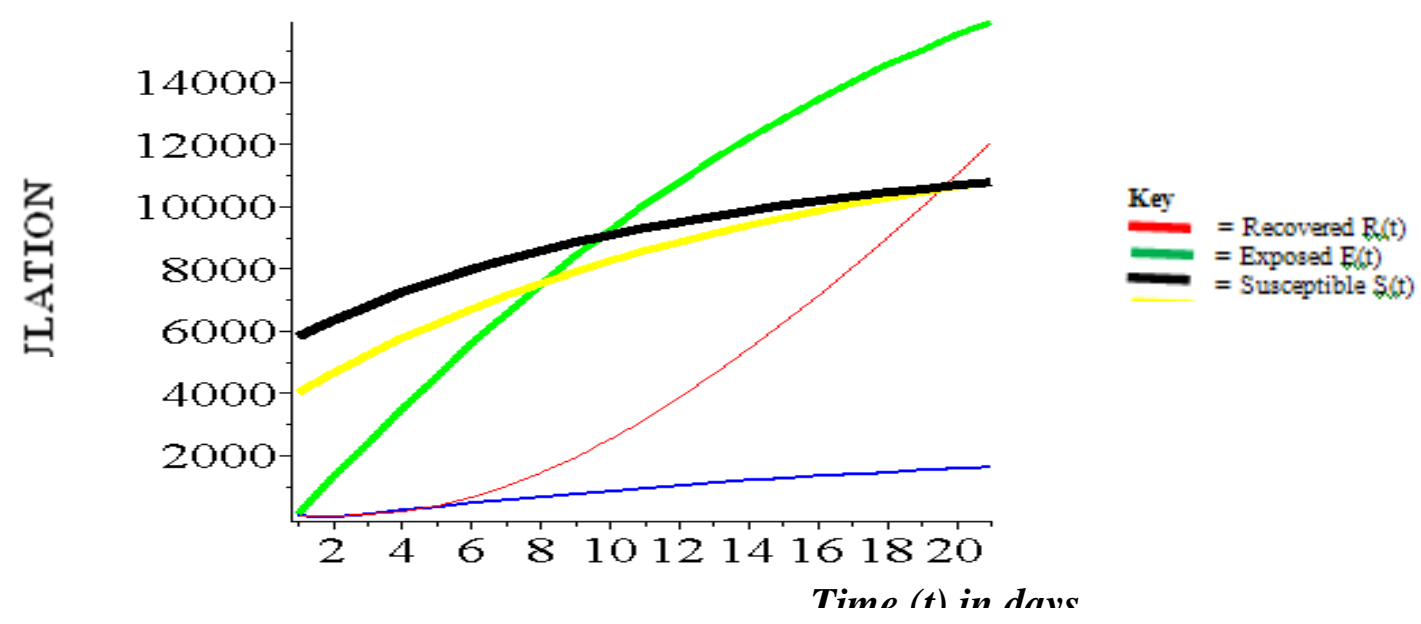

Figure 4: when $\mathrm{A}=200, \beta=0.2, \mathrm{~N}=0.02, \pi=2000, \delta=0.04, \sigma=0.09$, $\tau=0.8, \eta_{d}=0.9, \omega=0.1$ and $\rho=0.3$

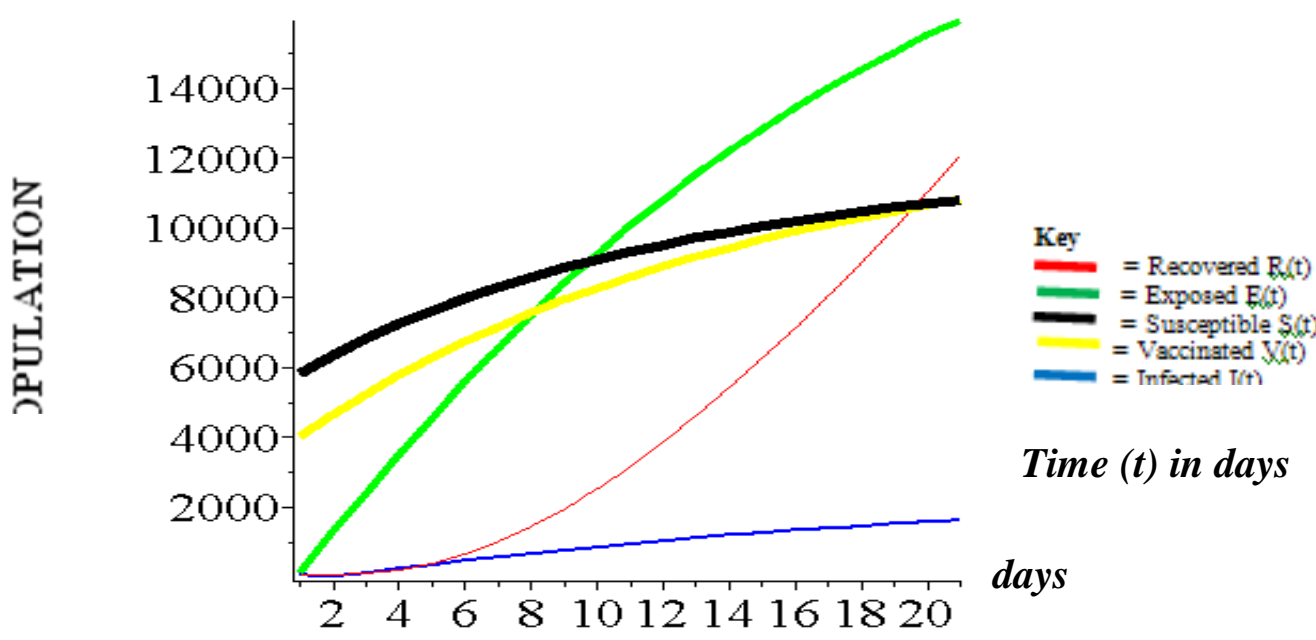

Figure 5: when $\mathbf{A}=300, \beta=0.2, \mathbf{N}=0.02, \pi=2000, \delta=0.04, \sigma=0.09$, $\tau=0.8, \eta_{d}=0.9, \omega=0.1$ and $\rho=0.3$ 
Mathematical Analysis Of Effect Of Area On The Dynamical Spread Of Measles

TABLE 1

\begin{tabular}{|c|c|c|c|c|c|c|}
\hline & $\begin{array}{c}\text { Time }(t) \text { in } \\
\text { days }\end{array}$ & $\begin{array}{c}\text { Susceptible } \\
\text { S (t) }\end{array}$ & $\begin{array}{c}\text { Vaccinated } \\
V(\mathrm{t})\end{array}$ & $\begin{array}{c}\text { Exposed } \\
\text { E (t) }\end{array}$ & $\begin{array}{l}\text { Infected } \\
\text { I (t) }\end{array}$ & $\begin{array}{c}\text { Recovered } \\
\mathrm{R}(\mathrm{t})\end{array}$ \\
\hline & 0 & 5800 & 4000 & 100 & 50 & 50 \\
\hline 1 & 0.1 & 6271.520335 & 2593.087043 & 1297.988830 & 22.66522106 & 87.78082640 \\
\hline 2 & 0.2 & 6718.303350 & 5153.725922 & 3438.973836 & 120.9093248 & 108.054041 \\
\hline 3 & 0.3 & 700.926740 & 5592.23625 & 3604.085427 & 236.9508916 & 207.159844 \\
\hline 4 & 0.4 & 7115.143696 & 5901.611624 & 4962.266560 & 360.3475078 & 397.445963 \\
\hline 5 & 0.5 & 7068.738118 & 6085.713794 & 8805.425189 & 497.7408976 & 685.2611298 \\
\hline 6 & 0.6 & 6884.478552 & 6052.1108612 & 7657.102472 & 637.26119151 & 1094.940321 \\
\hline 7 & 0.7 & 6593.265369 & 6034.600668 & 8978.811062 & 278.2909671 & 1568.480291 \\
\hline 8 & 0.8 & 628.928703 & 6042.293097 & 10228.64095 & 916.7278280 & 2164.613321 \\
\hline 9 & 0.9 & 5887.494554 & 5905.484602 & 11372.01806 & 1148.404573 & 2859.28413 \\
\hline 10 & 1.0 & 5444.258535 & 5747.831510 & 12383.33964 & 1169.598888 & 3644.89442 \\
\hline 11 & 1.1 & 5077.989405 & 5588.557841 & 13240.1087 & 1279.500813 & 4511.13652 \\
\hline 12 & 1.2 & 4755.168993 & 5441.114754 & 13968.30806 & 1370.51332 & 5445.91138 \\
\hline 13 & 1.3 & 4483.182625 & 5812.839625 & 14540.973112 & 1448.295776 & 6455.33837 \\
\hline 14 & 1.4 & 4262.240265 & 5206.668308 & 15000.3620 & 1311.604073 & 7466.77831 \\
\hline 15 & 1.5 & 4087.897965 & 5121.961808 & 15366.67804 & 1561.942984 & 8527.6864031 \\
\hline 16 & 1.6 & 3953.39265 & 5056.291443 & 15630.92748 & 1601.22548 & 9607.18465 \\
\hline 17 & 1.7 & 885.370500 & 5008.483232 & 15847.08749 & 1631.396171 & 10696.1343 \\
\hline 18 & 1.8 & 3774.938607 & 4969.329281 & 16003.18455 & 1654.327224 & 11382.1593 \\
\hline 19 & 1.9 & 3718.162555 & 4941.060392 & 16121.45786 & 1671.676993 & 12874.509 \\
\hline 20 & 2.0 & 3096.236098 & 4921.088885 & 16208.54162 & 1684.71183 & 13953.7985 \\
\hline
\end{tabular}


Mathematical Analysis Of Effect Of Area On The Dynamical Spread Of Measles

TABLE 2

\begin{tabular}{|c|c|c|c|c|c|c|}
\hline & $\begin{array}{c}\text { Time (t) } \\
\text { in days }\end{array}$ & $\begin{array}{c}\text { Susceptible } \\
\mathrm{S}(\mathrm{t})\end{array}$ & $\begin{array}{c}\text { Vaccinated } \\
\mathrm{V}(\mathrm{t})\end{array}$ & $\begin{array}{c}\text { Exposed } \\
\mathrm{E}(\mathrm{t})\end{array}$ & $\begin{array}{c}\text { Infected } \\
\mathrm{I}(\mathrm{t})\end{array}$ & $\begin{array}{c}\text { Recovered } \\
\mathrm{R}(\mathrm{t})\end{array}$ \\
\hline & 0. & 5800 & 4000 & 100 & 50 & 50 \\
\hline 1 & 0.1 & 6316.86462 & 4622.771029 & 1252.922656 & 22.46549259 & 87.77514150 \\
\hline 2 & 0.2 & 6784.613245 & 5200.202119 & 2380.0633056 & 116.9341504 & 107.738885 \\
\hline 3 & 0.3 & 7195.685297 & 5727.3193554 & 3488.224061 & 230.6755786 & 203.585442 \\
\hline 4 & 0.4 & 7533.718850 & 6206.270661 & 4571.985796 & 346.1474688 & 388.490820 \\
\hline 5 & 0.5 & 7863.962834 & 6640.372251 & 5625.063966 & 459.7921259 & 661.9114736 \\
\hline 6 & 0.6 & 8131.587253 & 7033.036420 & 6642.166903 & 570.4459598 & 1020.58139 \\
\hline 7 & 0.7 & 8361.406588 & 7387.574983 & 7619.173067 & 677.4530241 & 1460.37104 \\
\hline 8 & 0.8 & 8557.846721 & 7707.155916 & 8553.032763 & 780.3471722 & 1976.72960 \\
\hline 9 & 0.9 & 8724.951695 & 7994.787177 & 9441.637381 & 878.7875120 & 2564.82650 \\
\hline 10 & 1.0 & 8866.397017 & 8253.306918 & 10283.69473 & 972.5358023 & 3219.65933 \\
\hline 11 & 1.1 & 8985.504869 & 8485.377041 & 11078.61487 & 1061.441768 & 3936.13877 \\
\hline 12 & 1.2 & 9085.250963 & 8693.479793 & 11826.40590 & 1145.430717 & 4709.16009 \\
\hline 13 & 1.3 & 9168.333053 & 8879.917268 & 12527.57881 & 1224.492412 & 5533.66347 \\
\hline 14 & 1.4 & 9237.090954 & 9046.813575 & 13183.06093 & 1298.670946 & 6404.68414 \\
\hline 15 & 1.5 & 9293.627705 & 9196.119333 & 13794.11739 & 1368.055527 & 7317.38363 \\
\hline 16 & 1.6 & 9339.781455 & 9329.618074 & 14362.28063 & 1432.772132 & 8267.13266 \\
\hline 17 & 1.7 & 9377.157591 & 9448.934137 & 14889.28757 & 1492.975978 & 9249.0562 \\
\hline 18 & 1.8 & 9407.150674 & 9555.541647 & 15377.02415 & 1548.844819 & 10260.0562 \\
\hline 19 & 1.9 & 9430.965792 & 9650.774132 & 15827.47702 & 1600.573012 & 11294.9682 \\
\hline 20 & 2.0 & 9449.638994 & 9735.834654 & 16242.69198 & 1648.366342 & 12350.3863 \\
\hline
\end{tabular}


Mathematical Analysis Of Effect Of Area On The Dynamical Spread Of Measles

Table 3

\begin{tabular}{|c|c|c|c|c|c|c|}
\hline & $\begin{array}{c}\text { Time }(t) \text { in } \\
\text { days }\end{array}$ & $\begin{array}{c}\text { Susceptible } \\
S(t)\end{array}$ & $\begin{array}{c}\text { Vaccinated } \\
V(t)\end{array}$ & $\begin{array}{c}\text { Exposed } \\
E(t)\end{array}$ & $\begin{array}{l}\text { Infected } \\
I(t)\end{array}$ & $\begin{array}{c}\text { Recovered } \\
R(t)\end{array}$ \\
\hline & 0. & 5800 & 4000 & 100 & 50 & 50 \\
\hline 1 & 0.1 & 6321.400017 & 4625.740684 & 1248.414793 & 12.44551562 & 87.7749028 \\
\hline 2 & 0.2 & 6791.224118 & 5204.831304 & 2374.192482 & 116.5365948 & 107.707163 \\
\hline 3 & 0.3 & 7214.715633 & 5740.575979 & 3470.982892 & 230.0503001 & 203.228075 \\
\hline 4 & 0.4 & 7597.299301 & 6236.541303 & 4533.176076 & 344.4568101 & 387.598967 \\
\hline 5 & 0.5 & 7943.796440 & 6696.035642 & 5556.623761 & 456.0185470 & 659.606848 \\
\hline 6 & 0.6 & 8258.376971 & 7122.050072 & 6538.513161 & 563.7260289 & 1015.18642 \\
\hline 7 & 0.7 & 8544.636113 & 7517.281970 & 7477.125770 & 667.1638764 & 1449.57087 \\
\hline 8 & 0.8 & 8805.680001 & 7884.168785 & 8371.614558 & 766.1146305 & 1957.76460 \\
\hline 9 & 0.9 & 9044.200874 & 8224.919208 & 9221.817596 & 860.4699917 & 5234.70244 \\
\hline 10 & 1.0 & 9262.540355 & 8541.540133 & 10028.10555 & 950.1992794 & 3175.33697 \\
\hline 11 & 1.1 & 9462.742329 & 8835.859750 & 10791.25765 & 1035.330855 & 3874.69947 \\
\hline 12 & 1.2 & 9646.597130 & 9109.547369 & 11512.36113 & 1115.938262 & 4627.94499 \\
\hline 13 & 1.3 & 9815.678523 & 9364.130489 & 12192.72996 & 1192.129198 & 5430.38550 \\
\hline 14 & 1.4 & 9971.374733 & 9601.009573 & 12833.83931 & 1264.036636 & 6277.51376 \\
\hline 15 & 1.5 & 1011491452 & 9821.470892 & 13437.27307 & 1331.811664 & 7165.01954 \\
\hline 16 & 1.6 & 10247.38917 & 10026.69773 & 14004.68184 & 1395.617755 & 8088.80003 \\
\hline 17 & 1.7 & 10369.77100 & 10217.78020 & 14537.74970 & 1455.026161 & 9044.96530 \\
\hline 18 & 1.8 & 10482.92913 & 10395.72393 & 15038.16802 & 1512.012279 & 10029.8402 \\
\hline 19 & 1.9 & 10587.64277 & 10561.45765 & 15507.61502 & 1564.952776 & 11039.9632 \\
\hline 20 & 2.0 & 10684.61256 & 10715.84003 & 15947.74010 & 1614.623339 & 12072.0833 \\
\hline
\end{tabular}


Mathematical Analysis Of Effect Of Area On The Dynamical Spread Of Measles

Table 4

\begin{tabular}{|c|c|c|c|c|c|c|}
\hline & Time (t) in days & $\begin{array}{c}\text { Susceptible } \\
S(t)\end{array}$ & $\begin{array}{c}\text { Vaccinated } \\
V(t)\end{array}$ & $\begin{array}{c}\text { Exposed } \\
\text { E (t) }\end{array}$ & $\begin{array}{c}\text { Infected } \\
I(t)\end{array}$ & $\begin{array}{c}\text { Recovered } \\
R(t)\end{array}$ \\
\hline & 0 & 5800 & 4000 & 100 & 50 & 50 \\
\hline 1 & 0.1 & 6321.652004 & 4625.905671 & 1248.164349 & 22.44440577 & 87.7748729 \\
\hline 2 & 0.2 & 6791.591278 & 5205.088377 & 2373.866434 & 116.5145082 & 107.705401 \\
\hline 3 & 0.3 & 7215.770994 & 5741.311186 & 3470.026894 & 230.0155796 & 203.208222 \\
\hline 4 & 0.4 & 7599.718735 & 6238.221806 & 4531.021357 & 344.0363045 & 387.549440 \\
\hline 5 & 0.5 & 7948.232367 & 6699.128446 & 5552.820255 & 455.8090392 & 659.478779 \\
\hline 6 & 0.6 & 8265.428091 & 7127.000120 & 6532.747030 & 563.3525861 & 1014.88699 \\
\hline 7 & 0.7 & 8554.838258 & 7524.503461 & 7469.212447 & 666.5915212 & 1448.97098 \\
\hline 8 & 0.8 & 8819.503268 & 7894.040638 & 8361.485619 & 765.3218001 & 1956.71046 \\
\hline 9 & 0.9 & 9062.049692 & 8287.782638 & 9209.505759 & 859.4474367 & 2533.02655 \\
\hline 10 & 1.0 & 9284.755391 & 8557.697673 & 10013.72925 & 948.9405690 & 3172.86821 \\
\hline 11 & 1.1 & 9489.603634 & 8855.575355 & 10775.00595 & 1033.862789 & 3871.27178 \\
\hline 12 & 1.2 & 9678.328092 & 9133.047321 & 11494.47940 & 1114.271292 & 4623.40453 \\
\hline 13 & 1.3 & 9852.450319 & 9391.604889 & 12173.50666 & 1190.287922 & 5424.59653 \\
\hline 14 & 1.4 & 1001331102 & 9632.614246 & 12813.59405 & 1262.050392 & 6270.36309 \\
\hline 15 & 1.5 & 10162.09617 & 9857.32956 & 13416.34601 & 1329.713268 & 7136.41983 \\
\hline 16 & 1.6 & 10299.85893 & 10066.90435 & 13983.42448 & 1393.442366 & 8078.69199 \\
\hline 17 & 1.7 & 1042753801 & 10267.40138 & 14516.51693 & 1453.410295 & 9033.31901 \\
\hline 18 & 1.8 & 1054597313 & 10444.80131 & 1501731150 & 1509.792952 & 10016.6555 \\
\hline 19 & 1.9 & 1065591808 & 10615.01032 & 15487.47770 & 1562.766788 & 11025.2696 \\
\hline 20 & 2.0 & 10758.05175 & 10773.86681 & 15928.65179 & 1612.506681 & 12055.939 \\
\hline
\end{tabular}


Table 5

\begin{tabular}{|c|c|c|c|c|c|c|}
\hline & $\begin{array}{c}\text { Time }(t) \text { in } \\
\text { days }\end{array}$ & $\begin{array}{c}\text { Susceptible } \\
\mathrm{S}(\mathrm{t})\end{array}$ & $\begin{array}{c}\text { Vaccinated } \\
V(t)\end{array}$ & $\begin{array}{c}\text { Exposed } \\
E(t)\end{array}$ & $\begin{array}{c}\text { Infected } \\
\text { I (t) }\end{array}$ & $\begin{array}{c}\text { Recovered } \\
R(t)\end{array}$ \\
\hline & 0 & 5800 & 4000 & 100 & 50 & 50 \\
\hline 1 & 0.1 & 6321.735999 & 4625.960667 & 1248.080867 & 22.44403581 & 87.7748629 \\
\hline 2 & 0.2 & 6791.713663 & 52051.17407 & 2373.757754 & 116.5071459 & 107.704813 \\
\hline 3 & 0.3 & 7216.122737 & 5741.556228 & 3469.708274 & 230.0040064 & 203.201604 \\
\hline 4 & 0.4 & 7600.525173 & 6238.781948 & 4530.303152 & 344.3317936 & 387.532931 \\
\hline 5 & 0.5 & 7949.711025 & 6700.159393 & 5551.552394 & 455.7392064 & 659.436092 \\
\hline 6 & 0.6 & 8267.778627 & 7128.650248 & 6530.824819 & 563.2281029 & 1014.78712 \\
\hline 7 & 0.7 & 8558.239480 & 7526.910975 & 7466.574190 & 666.4007200 & 1448.77102 \\
\hline 8 & 0.8 & 8824.112242 & 7897.332100 & 8358.108195 & 765.0574772 & 1956.35907 \\
\hline 9 & 0.9 & 9068.001808 & 8242.072194 & 9205.399602 & 859.1064762 & 2532.46787 \\
\hline 10 & 1.0 & 9292.165021 & 8563.086747 & 10008.93322 & 948.5314482 & 3172.04515 \\
\hline 11 & 1.1 & 9498.565185 & 8862.152690 & 10769.58230 & 1033.373045 & 3870.12889 \\
\hline 12 & 1.2 & 9688.917319 & 9140.889279 & 11488.50907 & 1113.715000 & 4621.89041 \\
\hline 13 & 1.3 & 9864.725785 & 9400.775942 & 12167.08489 & 1189.673190 & 5422.66573 \\
\hline 14 & 1.4 & 10027.31563 & 9643.167596 & 12806.82648 & 1261.386910 & 6267.97761 \\
\hline 15 & 1.5 & 10177.85873 & 9869.307841 & 13409.34516 & 1329.011880 & 7153.55024 \\
\hline 16 & 1.6 & 10317.39565 & 10080.34038 & 13976.30671 & 1392.714698 & 8075.31813 \\
\hline 17 & 1.7 & 10446.85398 & 10277.31895 & 14509.39988 & 1452.668436 & 9029.43043 \\
\hline 18 & 1.8 & 10567.06369 & 10461.21598 & 15010.31186 & 1509.049167 & 10012.2516 \\
\hline 19 & 1.9 & 10678.77012 & 10632.93010 & 15480.70943 & 1562.033290 & 11020.3597 \\
\hline 20 & 2.0 & 10782.64486 & 10793.29303 & 15922.22465 & 1611.795426 & 12050.5418 \\
\hline
\end{tabular}

\section{DICUSION OF RESULT}

The SVEIR model was considered to gain more insight into the effect of Habitat area on dynamic spread of measles. This Habitat area plays a crucial role in the control of spread of measles virus in the environment. It is observed from the results above that the higher the Habitat area, the lower will be spread of this measles virus and the higher will be the recovery rate. Also considered the possibility of measles outbreak in a community when certain threshold quantity, the basic reproduction number $\left(R_{0}\right)$ exceeded unity (one).

We considered basic reproduction number which is an important tools for public health officials to determined the epidemic state of any deadly disease in a community. Using this threshold, we discovered that when the habitat area of measles patient is highly increased, the lesser would be the number of infected individuals and higher would be the number of recovered people. Conversely, when habitat area was low, for instance at $\mathrm{A}=1$ (Fig. 1), the $R_{0}$ is greater than one. This implies that there would be high level of epidemic within few days because of congestion of the area and the population of recovered people rise after six days. 
Theoretically, our results are based on the fact that the diseases free equilibrium (DFE) point is locally stable whenever the threshold quantity $R_{0}<1$ and unstable when $R_{0}<1$ (see lemma 1 ). We also showed that whenever the associated reproduction number $R_{0}^{\infty}<1$ in a community, there would be endemic situation of measles in that community unless there is quick intervention to keep the basic reproduction number less than unity.

\section{REFERENCES}

[1] Adeoye I.A, Dairo M.D, Adedokun H.O, and Makanjuola J, (2010): Investigation of a meales outbreak in a Rural Nigerian community - The Aladura experience. African journal of Microbiology Research Vol. 4(5), pp. 360-366.

[2] Adewale S.O, Plodder C.N, and Gumel A.B (2009): Mathematical Analysis of a TB Transmission Model with DOTS. Canadian Applied Mathematical Quarterly Volume 17, number 1, spring 2009.

[3] Adewale S.O. Olarenwaju P. O. Taiwo S.S. Anake T.A. and Famewo M.M. (2012): Mathematical Analysis of the effect inmmunization on the dynamical spread of measles.

[4] Annual Report of the National Diseases Surveillance Centre, 2000. Dublin, Health Protection Surveillance Centre; 2001.ISSN: 1649. Available from:

http://www.hpsc.ie/hpsc/ AboutHPSC/ Annual Reports/File, 520, en.pdf.

[5] Ca ceres VM, Strebel PM, Sutter RW (2000): Factors determining prevalence of material antibody to measles virus throughout infancy: a review. Clin Infact Dis 2000; 31: 110-19.

[6] Carabin H, Edumunds WJ, Kou U, van den Hof S \& Nguyen VH (200). The average cost of measles cases and adverse events following vaccination in industrialized countries. BMC Public Health 2:22 (http://www.biomedcentral.com/1471-2458/2/22).

BMC Public Health 2:22 (http://www.biomedcentral.com/1471-2458/2/22)

[7] Dan Long ${ }^{1}$ and Zhongyi Xiang (2011): On the study of an SEIV epidermic model concerning vaccination and vertical transmission.

[8] Ousmane MOUSSA TESSA (2006): Mathematcial model for control of measles by vaccination

[9] Strebel P, Cochi S, Grabowsky M (2003): The unfinished measles immunization agenda. J Infect Dis 2003; 187 (Suppl 1): SI-S7.

[10] World Health Organization, (2004): Measles vaccines: WHO position paper. Wkly Epidemiol. Rec. 79, 130-142.

[11] World Health Organization (2009): Measles Fact Sheet No 286 Geneva Switzerland: www.who.int/mediacentre/factsheets/fs286/en.

[12] World Health Organization (2011): WHO Vaccine Preventable Diseases: Monitoring System 2011 Global Summary, available at: http://www.who.int/immunization_monitoring/en/globalsummary/timeseries/tsincidence mea.htm.

[13] Robet M.G and Heesterbeek J.A.P: Mathematical model in Epidemiology. VolumeIII. Encyclopedia of life support system. 